\title{
Magnetic Field Distributions inside Magnetically Driven Nanofluids for Thermal Management of CPUs
}

\author{
Serkan Doganay ${ }^{1,2, *}$, Levent Cetin ${ }^{2}$, Mehmet Akif Ezan ${ }^{3}$ and Alpaslan Turgut ${ }^{3}$ \\ ${ }^{1}$ Dokuz Eylül University, The Graduate School of Natural and Applied Sciences, İzmir, Turkey, \\ ${ }^{2}$ İzmir Kâtip Çelebi University, Department of Mechatronics Engineering, İzmir, Turkey, \\ ${ }^{3}$ Dokuz Eylül University, Department of Mechanical Engineering, İzmir, Turkey,
}

\begin{abstract}
Herein a magnetic actuation strategy is proposed to manipulate magnetic nanofluids in CPU thermal management applications. The proposed concept consists of a multi-circular microchannel unit and a rotating permanent magnet. A set of numerical simulations have been carried out to evaluate the influences of (i) magnet diameter, (ii) distance between the magnet and the microchannel, and (iii) shape of the magnet on magnetic flux densities (MFD) inside microchannels with $\mathrm{Fe}_{3} \mathrm{O}_{4}$ magnetic nanofluid. The results indicated that the MFD on the magnetic nanofluid significantly varies along with the radial position within the microchannel.
\end{abstract}

\section{Introduction}

The advancement in sophisticated engineering designs has expedited the development of micro and nanoscale electronic components, which brings a remarkable enhancement in the speed and performance of personal computers (PCs), laptops, tablets, and cell phones by increasing the frequency of a CPU. Increasing the performance of CPUs causes a high heat dissipation on CPU from relatively small and narrow surface areas. The accumulation of the dissipated heat is still a significant challenge in computer science and technology as inadequately designed thermal management solutions which causes to overheating of CPUs [1]. The heat removal from the CPU is generally performed by air, liquid, and thermoelectric cooling [2]. Conventional aircooling systems are not able to handle high heat loads [3]. On the other hand, liquid-cooling systems are more promising for convective heat transfer by micro-sized channels. In order to enhance the performance of the liquid coolers, it is possible to use new generation high conductive fluids. Nanofluids are the colloids that consist of a base fluid, e.g., water, ethylene glycol and oil, and nano-sized particles, e.g., metals, metal oxides, and carbon nanotubes. They are promising for liquid CPU coolers with the enhanced cooling performance [4]. It is also possible to use superparamagnetic iron oxide nanoparticles (SPIONs) in such colloids. Then, the colloid is named as magnetic nanofluid. Magnetic nanofluids have also higher conductivity while it is compared to the conventional base fluids [5]. These fluids can be manipulated under the influence of the external magnetic field. Moreover, their thermophysical properties can be tuned when they exposed to an external magnetic field [6]. Therefore, magnetic nanofluids can be actuated as an alternative pumping method in microchannel flow for thermal engineering systems [7].

Magnetic actuation can be provided for magnetic nanofluids by either permanent magnets or electromagnets. The generated magnetic field strength can be adjusted by controlling the current through the electromagnet. However, there will be an additional heat generation due to the current through the coils. This makes them unsuitable when they are used for magnetic actuation for CPU cooling applications. On the other hand, permanent magnets can generate a high magnetic field gradient in small volumes with the continuous expenditure of energy [8]. Since they are naturally magnetized, there will be no heat generation, which makes them compatible and effective for CPU cooling applications. Also, the permanent magnets can be translated or rotated for the magnetic actuation with lowlevel noise operation by micro motors in relatively small volumes compared to other types of pumps.

Even though the actuation of the magnetic nanofluids by permanent magnets for CPU coolers has an important potential, there are limited applications available in the literature. One of the main reasons behind this is the lack of knowledge of the MFD characteristics of magnetically driven nanofluids within microchannels. Herein, we present a set of numerical analyses as a preliminary investigation on the behavior of the magnetic field generated by a permanent magnet on the $\mathrm{Fe}_{3} \mathrm{O}_{4}$-water magnetic nanofluid in the microchannel unit. The influences of the magnet diameter, the distance between the magnet and microchannel region, and the shape of the magnet on the MFD distributions are evaluated. The geometry of the microchannel unit is designed considering the dimensions of a commercially available CPU.

Corresponding author: serkan.doganay@outlook.com 


\section{Proposed Actuation Strategy}

Figure 1a shows the proposed actuation strategy. A microchannel unit is placed underneath the CPU to reject the excessive heat and provide thermal control. In order to provide the magnetic nanofluid flow within the microchannel unit, a magnet rotates along the circular path and generates a spatial magnetic field gradient. Herein a permanent magnet is placed on a rotor under the microchannel, and an electric motor drives the rotor to maintain the rotational motion. The microchannel unit consists of circular multi microchannels in the angular range of $0-270^{\circ}$. The details of the microchannel unit and magnet position are shown in Figure 1b. Circular microchannel geometry is designed based on dimensions of a commercial CPU (Intel's ${ }^{\circledR}$ 4th generation Core ${ }^{\mathrm{TM}}$ i7-10510U processor) [12]. The dimensions of the microchannel unit are provided in Table 1.
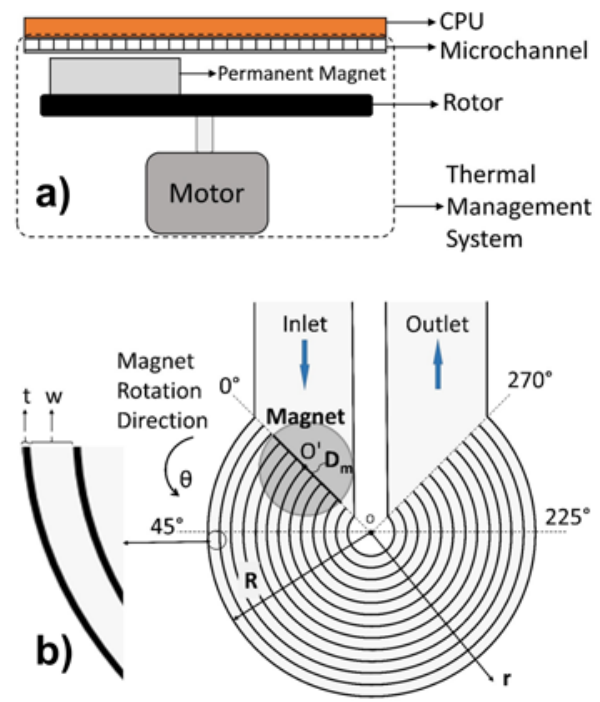

Fig. 1. a) An overview of the assembly, b) Close look to the microchannel unit and magnet position.

Table 1. Dimensions of the microchannel unit.

\begin{tabular}{ll}
\hline Parameter & Value \\
\hline Outer Radius of the microchannel, $R$ & $13 \mathrm{~mm}$ \\
The thickness of the microchannel wall, $t$ & $900 \mu \mathrm{m}$ \\
The width of the microchannel, $w$ & $100 \mu \mathrm{m}$
\end{tabular}

Within the proposed actuation strategy, the magnetic nanofluid flow is occurred due to the magnetic force generated by the permanent magnet. Permanent magnets are non-uniform magnetic field sources, which makes them suitable for magnetic force generation in small structures. In the absence of the electric field, the magnetic force can be expressed as [9]:

$$
\mathbf{F}_{\mathbf{m}}=(\mathbf{M} \cdot \nabla) \mathbf{B}
$$

where $M$ is the magnetization vector, and $B$ is the MFD. It means that when a magnetic field gradient is available there will be a magnetic force generated. Magnetic nanofluids can behave as a single-phase medium if the superparamagnetic nanoparticles are well dispersed [10]. In that case, the body force acting on a magnetic nanofluid can be calculated by [11]:

$$
\mathbf{F}_{\mathbf{m}}=\frac{\forall \Delta \chi}{\mu_{0}}(\mathbf{B} \cdot \nabla) \mathbf{B}
$$

where $\forall$ is the magnetizable volume, $\mu_{0}$ is the permeability of the free space, $\Delta \chi$ is the susceptibility difference between particles and base fluid.

\section{Numerical Model}

A 3D numerical model was developed in COMSOL software to simulate the MFD distributions within the microchannel unit when the permanent magnet is stationary at $\theta=0^{\circ}$. The magnet and the circular multi microchannels were placed in a cubic air domain with an edge length of $100 \mathrm{~mm}$. It is assumed that magnetic flux density is conserved in all domains. The microchannels are filled with $\mathrm{Fe}_{3} \mathrm{O}_{4}$-water magnetic nanofluid (4.8 vol. \%). The external magnetic field $(\mathbf{H})$ by the permanent magnet is evaluated by resolving the following Gauss’ Law:

$$
\nabla \cdot\left(\mu_{0} \mu_{r} \mathbf{H}+\mathbf{B}_{\mathbf{r}}\right)=0
$$

Where $\mathbf{B}_{\mathbf{r}}$ is the remanence and $\mu_{\mathrm{r}}$ is the relative permeability (1.05) of the magnet. In the numerical model, a NdFeB permanent magnet was employed with remanence and relative permeability of $1.3 \mathrm{~T}$ and 1.05 , respectively. Then, the MFD is determined by $\mathbf{B}=\mu_{0} \mathbf{H}(1+\chi)$ where $\chi$ is the magnetic susceptibility of the magnetic nanofluid. The susceptibility of the magnetic nanofluid determined experimentally after magnetic properties measurement by vibrating sample magnetometer (VSM) as 0.22 . The free tetrahedral mesh structure was used with 7953077 elements. The convergence criterion is defined as $10^{-4}$. Before the numerical simulations, the numerical model of Ozbey et al. [13] has been verified with an MFD deviation below $5 \%$. Notice that the driving force of the magnetic nanofluid within the circular microchannel arises from the tangential component of the MFD. That is variations of the tangential component of the MFD are given along the radial and angular positions within the microchannel unit.

\section{Results and Discussions}

\subsection{Influence of the Magnet Diameter}

Magnet diameter is varied from $6 \mathrm{~mm}$ to $12 \mathrm{~mm}$ with a constant magnet thickness of $4 \mathrm{~mm}$. Then, MFD distributions within the microchannel are evaluated. In Figure 2a, b, and c, the variations in the tangential component of the MFD at different radial positions are given along the angular position. 

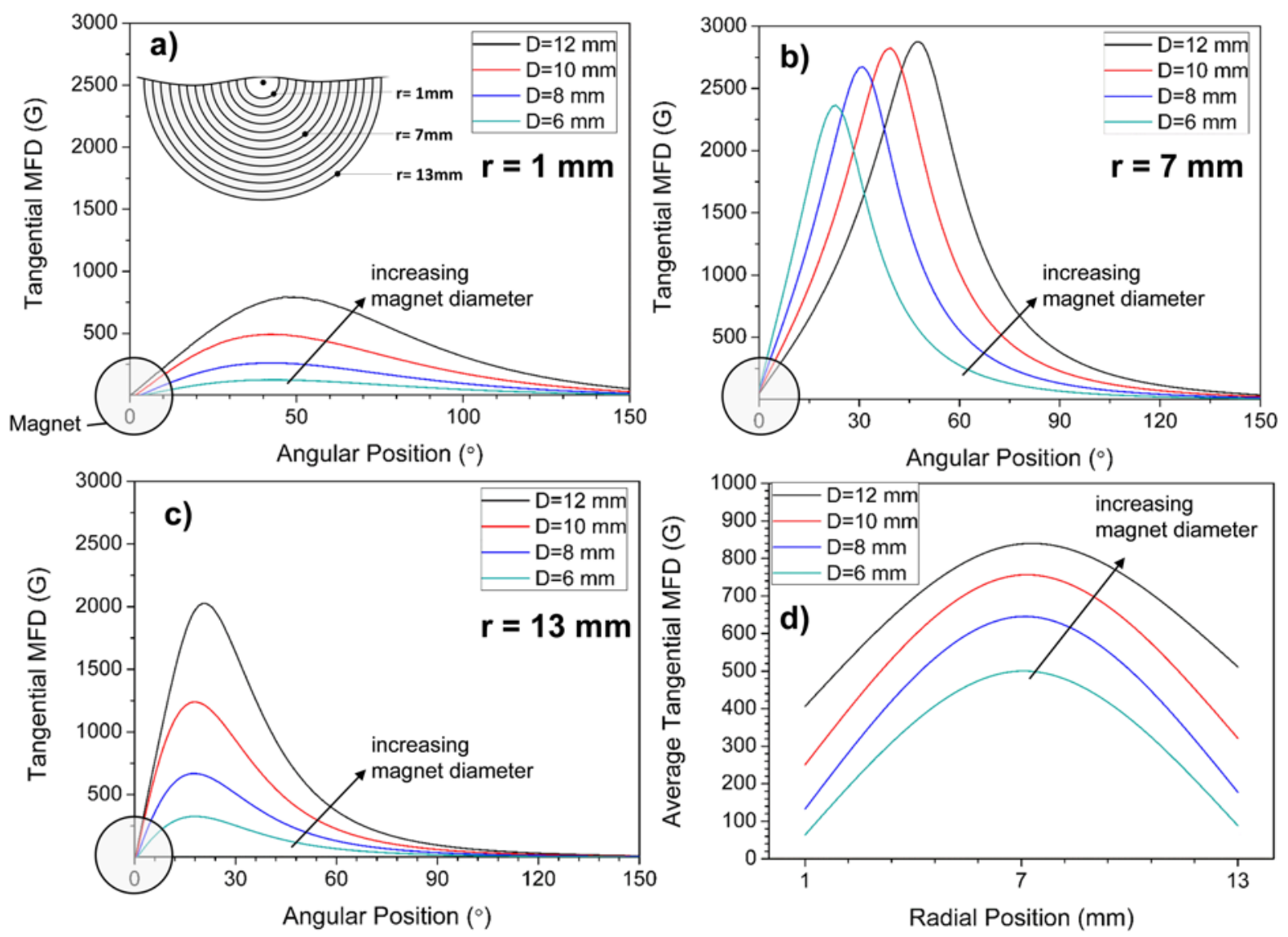

Fig. 2. Variations of the tangential component of MFD inside the magnetic nanofluid wrt angular position for different magnet diameters of a) $r=1 \mathrm{~mm}, \mathrm{~b}$ ) $\mathrm{r}=7 \mathrm{~mm}, \mathrm{c}) \mathrm{r}=13 \mathrm{~mm}$ and d) average tangential MFD with respect to radial position.

Three different radial positions of $\mathrm{r}=1 \mathrm{~mm}, 7 \mathrm{~mm}$, and $13 \mathrm{~mm}$ are considered since they represent the innermost, outer-most, and middle channels of the multichannel unit. In the current simulations, the distance between the magnet and the microchannel unit is kept constant at $0.5 \mathrm{~mm}$. It is clearly seen that the magnitude of the MFD significantly decreases with decreasing the magnet diameter. Regardless of the radial position, the highest MFD values are obtained at the center of the microchannels, and the lowest MFD values are observed at $\mathrm{r}=1 \mathrm{~mm}$.

It is also observed that the angular positions of the highest MFD values are very close to each other for all magnets at $\mathrm{r}=1$ and $13 \mathrm{~mm}$. However, this behavior changes at $r=7 \mathrm{~mm}$. Moreover, the angular positions of the highest MFD values are closer to the entrance (around $\sim 20^{\circ}$ ) at $\mathrm{r}=13 \mathrm{~mm}$, and the position is shifted through $\sim 50^{\circ}$ at $\mathrm{r}=1$ and $7 \mathrm{~mm}$. Figure 2d, on the other hand, demonstrates that the mean MFD is higher at the center of the circular multi microchannel, and the values at the borders are almost identical. The ratios between max. and min. values in Figure 2d are 2.1, 3, 4.9 and 7.8 for $\mathrm{D}=12$, 10,8 and $6 \mathrm{~mm}$, respectively. These behaviors are directly related to the geometry of the magnets and microchannel structure. The MFD generated by the magnets directly depends on the normal distance from the magnet center. Since the normal distances from the magnet center to the microchannel borders are similar for each magnet, the mean MFD acts on radial positions of $r$
$=1$ and $13 \mathrm{~mm}$ should be also identical. However, in the circular multi microchannel structure, a constant normal distance from the center of the magnet corresponds to a higher angular position for inner channels. Therefore, the angular position of the highest MFDs vary.

\subsection{Influence of the Magnet Distance}

Four different magnet distances of $0.5,1.5,2.5$, and 3.5 $\mathrm{mm}$ to microchannel unit were utilized in order to examine the influence of the distance at a constant magnet diameter of $12 \mathrm{~mm}$ and thickness of $4 \mathrm{~mm}$. It is seen in Figure 3a, b, and c that the MFD becomes lower with increasing the distance as expected. Apart from the angular position where the max. MFD has occurred for $r$ $=7 \mathrm{~mm}$, the distance influence on the MFD behavior is very close to the behavior observed in the diameter influence. At the center, the angular positions of the highest MFDs are almost similar for all magnet distances. This is related to the there is no change in the direction of normal vector from any point on the magnet surface to the center of the circular multi microchannel. In terms of the mean MFD, again the average MFD values are similar in the borders, and it is maximum at the center (Figure 2d). However, this time, the ratios between highest and lowest average values do not change with varying distance and found as $2.1,2.3,2.4$, and 2.5 for distance of $0.5,1.5,2.5$, and $3.5 \mathrm{~mm}$, respectively. 

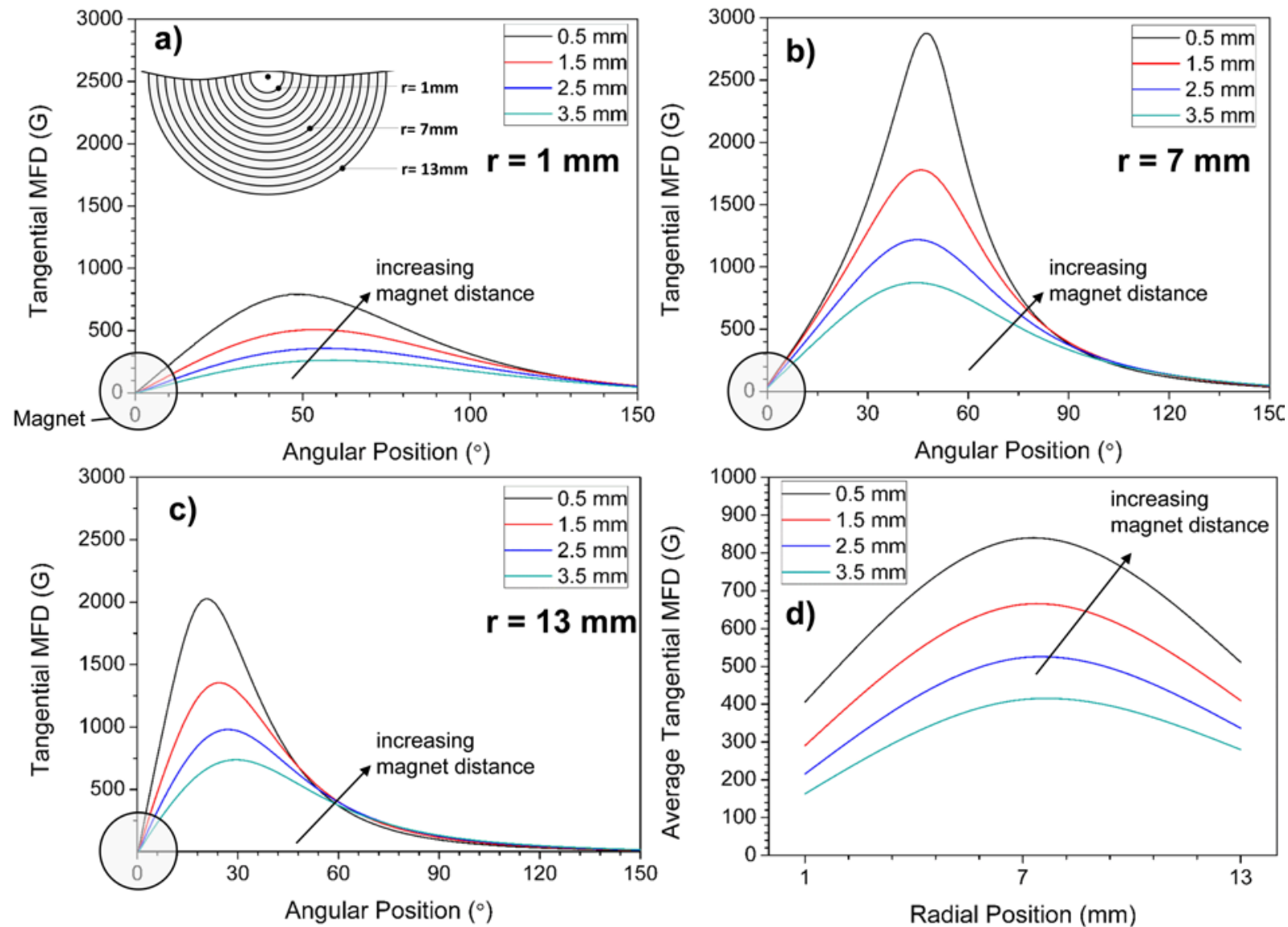

Fig. 3. Variations of the tangential component of MFD inside the magnetic nanofluid wrt angular position for different magnet distances at a) $r=1 \mathrm{~mm}, \mathrm{~b}$ ) $\mathrm{r}=7 \mathrm{~mm}, \mathrm{c}) \mathrm{r}=13 \mathrm{~mm}$ and d) average tangential MFD with respect to radial position.
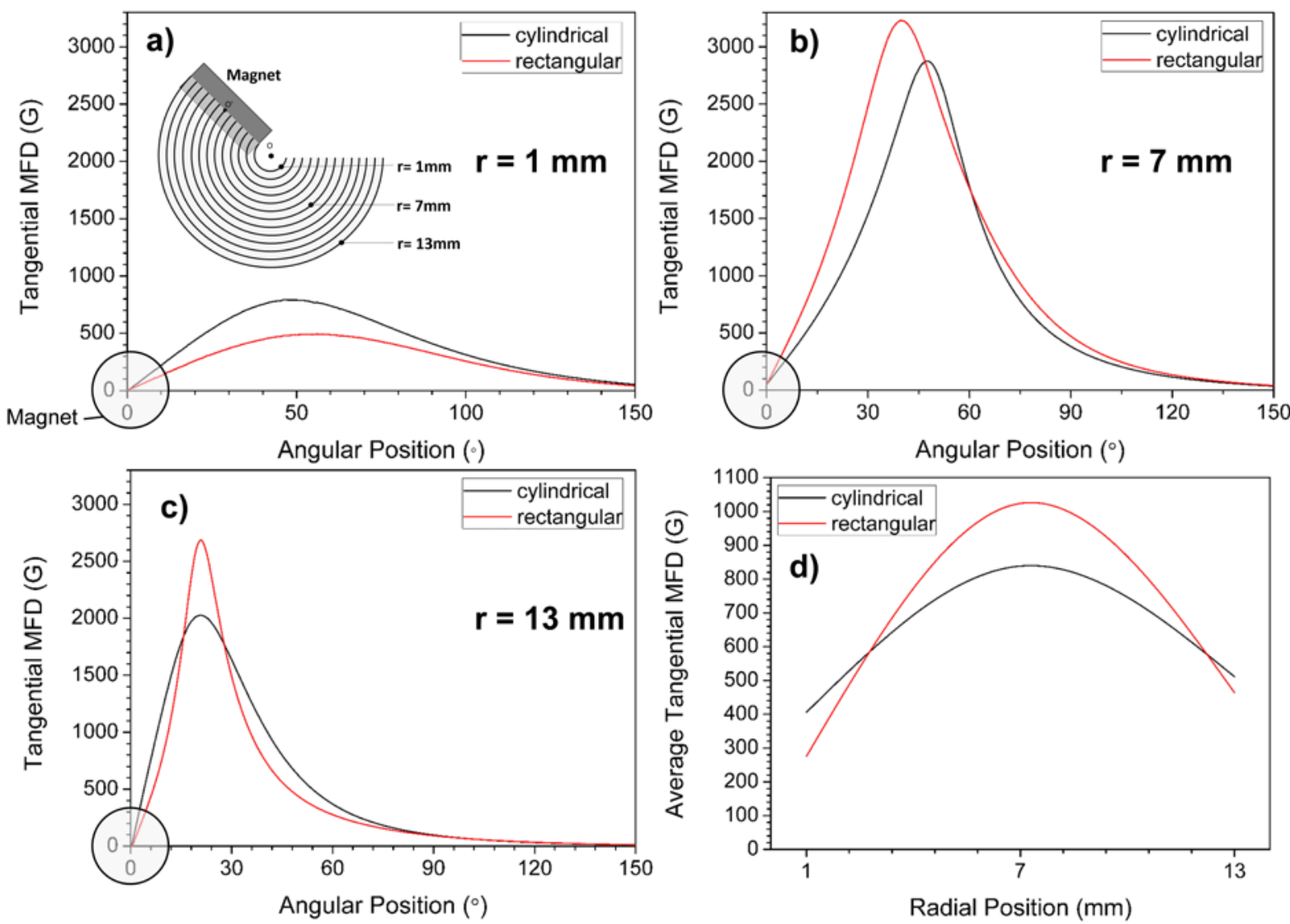

Fig. 4. Variations of the tangential component of MFD inside the magnetic nanofluid wrt angular position for different magnet shapes at a) $r=1 \mathrm{~mm}$, b) $r=7 \mathrm{~mm}, \mathrm{c}) \mathrm{r}=13 \mathrm{~mm}$ and d) average tangential MFD with respect to radial position. 


\subsection{Influence of the Magnet Shape}

A rectangular magnet $(9.5 \times 12 \times 4 \mathrm{~mm})$, which has a similar volume with the cylindrical magnet (D12x4 mm) was employed for investigating the shape influence. Figure $4 \mathrm{a}, \mathrm{b}$, and $\mathrm{c}$ indicate that the maximum MFD is higher at $r=7 \mathrm{~mm}$ and $r=13 \mathrm{~mm}$ for rectangular, and $r$ $=1 \mathrm{~mm}$ for cylindrical magnet. Due to the geometry of rectangular magnet, the inner border is exposed to a lower amount of magnetic field lines, like it is placed in a dead-zone. This causes a decrement in MFD at $r=1$ when compared with cylindrical magnet. Figure 4d depicts that the maximum average MFD value is higher for rectangular magnet at the center. However, at the borders an adverse behavior is observed. It is obvious for $r=1 \mathrm{~mm}$, due to the lower MFD. To explain the lower avg. MFD at $r=13$ for rectangular magnet, one should notice from Figure $3 c$ that the MFD generated by rectangular magnet acts on smaller angular area than the rectangular magnet. In terms of the change in MFD between center and borders, the ratios between maximum and minimum average values are found as 2.1 and 3.7 for cylindrical and rectangular magnets, respectively.

\section{Conclusions}

In this preliminary study, a magnetic actuation strategy for magnetic nanofluids is introduced by a permanent magnet on a microchannel unit which can be used for cooling of CPUs. For this purpose, the MFD distribution in the channel region is investigated in terms of diameter, distance, and shape influence. Following conclusions can be drawn:

- Increasing the magnet diameter increases the MFD on the magnetic nanofluid inside the microchannel unit. Moreover, the ratio between the average MFD on the center and the borders are lower for higher magnet diameter.

- Increasing the distance between the magnet and the channel drastically decreases the MFD on the microchannel unit when the range is increased. However, increasing the distance has almost no influence on the ratio between the avg. MFD on the center and the border. - Higher MFD values can be obtained by using a rectangular magnet rather than using the cylindrical one. However, the implementation of a rectangular magnet causes a higher MFD difference between center and border.

\section{Acknowledgements}

This article is based upon work from COST Action NANOUPTAKE CA15119, supported by COST (European Cooperation in Science and Technology). Also, the author SD would like to gratefully acknowledge the financial support provided by EU COST Action CA15119: Overcoming Barriers to Nanofluids Market Uptake (NANOUPTAKE) in the scope of ITC Conference Grants in order to attend ICPEME 2020.

\section{References}

1. M.M. Sarafraz, A. Arya, F. Hormozi, V. Nikkhah, Appl. Ther. Eng., 112, 1373-1381 (2017)

2. X.C. Tong, Advanced Materials for Thermal Management of Electronics Packaging (Springer Science+Business Media, New York, 2011)

3. V. Singhal, S.V. Garimella, A. Raman, Appl. Mech. Rev., 57, 3 (2004)

4. M.H. Al-Rashed, G. Dzido, M. Korpys, J. Smolka, J. Wojcik, Microelect. Rel., 63, 159165 (2016)

5. S. Doganay, R. Alsangur, A. Turgut, Mater. Res. Express 6, 11 (2019)

6. P.D. Shima, J. Philip, B. Raj, Appl. Phys. Lett., 95, 13 (2009)

7. B. M'Hamed, N.A.C. Sidik, M. Yazid, R. Mamat, G. Najafi, G.H.R. Kefayati, Int. Commun. Heat Mass, 78, 60-67 (2016)

8. J.M.D. Coey, J Magn. Magn. Mater., 248, 441456 (2002)

9. M.C. Weston, M.D. Gerner, I. Fritsch, Anal. Chem., 82, 3411-3418 (2010)

10. N.T. Nguyen, Microfluid. Nanofluid., 12, 1-16 (2012)

11. N. Pamme, Lab. Chip., 6, 24-38 (2006)

12. 10th Generation Intel ${ }^{\circledR}$ Core $^{\mathrm{TM}}$ Processors Datasheet, Intel Corp., September 2019

13. Ozbey, M. Karimzadehkhouei, S.E. Yalcin, D. Gozuacik, A. Kosar, Microfluid. Nanofluid., 18, 447-460 (2015) 